\title{
STABILITY OF THE RF SYSTEM AT THE SPring-8 LINAC
}

\author{
T. Asaka*, H. Hanaki, T. Hori, T. Kobayashi, A. Mizuno, H. Sakaki, \\ S. Suzuki, T. Taniuchi, K. Yanagida, H. Yokomizo, H. Yoshikawa \\ SPring-8, Mihara, Mikazuki, Sayo-gun, Hyogo 678-5198 Japan
}

\section{Abstract}

The stability of the beam energy and energy spread at both the $1-\mathrm{GeV}$ S-band linac and the $8-\mathrm{GeV}$ synchrotron ring are crucial factors for determining the injection time for the storage ring. Since the linac has a lot of high-power RF equipment, any drift of the output power and phase from the various type of RF equipment will affect the beam energy in beam operation. We measured the output power and phase stability for many types of RF equipment.

In order to investigate the drift of the RF parameters in the klystrons, which can be influenced by outside factors, the cooling water temperature and the environmental temperature were also measured. It turns out that the phase of klystrons coincide with the cooling water temperature drift. Although controlled by an air conditioning, the room temperature can vary about $4.0^{\circ} \mathrm{C}$ in the course of a day, affecting the high-power klystron drive system.

After improvements of the air conditioning control system and the cooling water system for the klystron cavities, a beam current stability was reduced to $0.7 \%(1 \sigma)$ from $9.0 \%(1 \sigma)$ in beam operation.

\section{INTRODUCTION}

The 1-GeV linac of SPring-8 consists of a thermionic gun, a bunching system and 26 accelerating structure columns. The linac is able to produce three kinds of the beam pulse widths (1 nsec, 10-40 nsec and $1 \mu \mathrm{sec})$ that are requested by the storage ring operation mode; single bunch operation and multi bunch operation. In normal beam operation at two-week or three-week intervals, the beam is injected into the storage ring two times a day. In order to realize uniformity of the bunch train in the storage ring, it has to satisfy the requirements of both the reproduction and stabilization of beam energy at the injector linac, which has a lot of highpower RF equipment. The energy stability as well as the beam change transmission has been a very important issue for stable synchrotron ring injection since the operation of the synchrotron ring began in December 1996. In order to investigate the stability of the beam current and energy at the $1-\mathrm{GeV}$ linac-synchrotron beam transport (LSBT) as illustrated in Fig. 1, the beam current was measured by a wall current monitor placed on the $1-\mathrm{GeV}$ straight line and after the $1-\mathrm{GeV}$ bending magnet while using a beam slit to permit an energy spread of $1.0 \%$. The result of this measurement is shown in Fig. 2. Though there was no change in operating conditions during the measurement, the beam

\footnotetext{
*Email: asaka@spring8.or.jp
}

trigger was stopped six times for such reasons as the vacuum deterioration at the RF power line and excessive current of the klystron modulator. It was observed that the drift of the beam current had a period of 25 minutes at the LSBT. In addition, beam drift for the 10 hours was also observed. The following are considered drift factors: change in vacuum pressure, the high-power klystron drive system that depends on the environmental temperature, and the resonant frequency of klystron cavities that depend on the cooling water temperature. Furthermore, the shot-by-shot center energy fluctuation was expected to be caused by PFN voltage fluctuation of the 13-set klystron modulator, along with the jitter of the modulator and thyratron triggers.

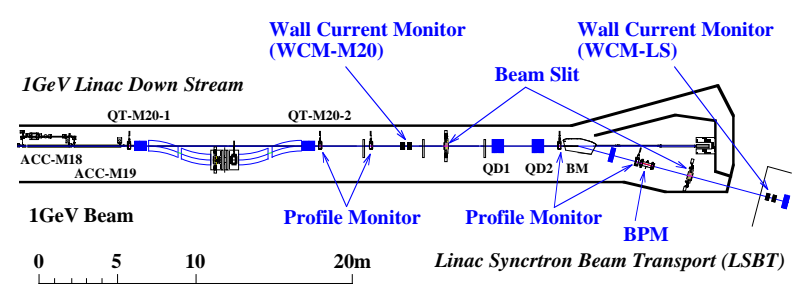

Figure 1: Layout of linac-synchrotron beam transport.

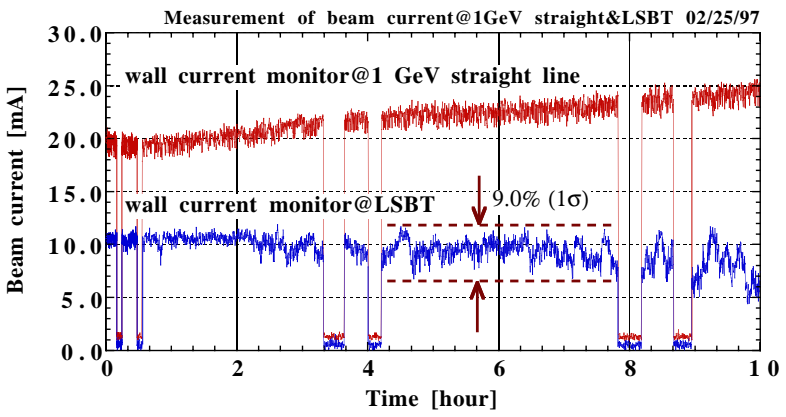

Figure 2: Drift of beam current at $1-\mathrm{GeV}$ straight line and after $1-\mathrm{GeV}$ bending magnet using a beam slit to permit energy spread of $1.0 \%$.

\section{MEASUREMENT AND ANALYSIS}

\section{$2.1 \quad$ RF system}

The schematic of the RF system for the linac is shown in Fig. 3. The RF system consists of a $7 \mathrm{MW}$ booster klystron (MELCO PV2012) drive system, another highpower klystron drive system, and a 13-set $80 \mathrm{MW}$ klystron (TOSHIBA E3712). Each klystron feeds into two 3-m long accelerating structures with the exception of the H0 


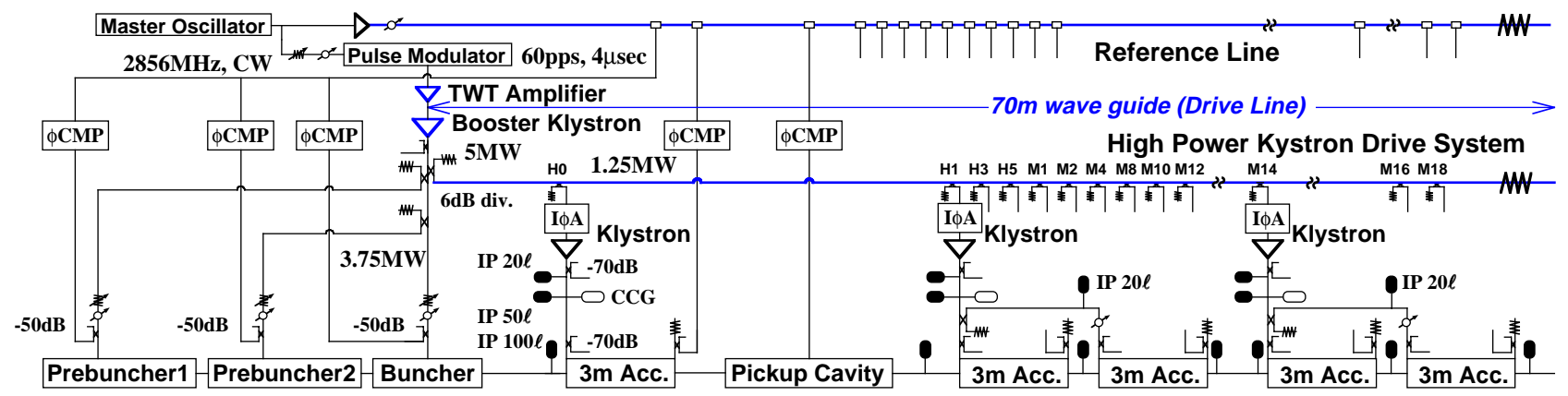

Figure 3: Schematic of RF composition.

klystron, which feeds into a 3-m long accelerating structure at the $60 \mathrm{MeV}$ pre-injector. In addition, a reference line is provided for the phase measurement and feedback system [1].

The $2856 \mathrm{MHz} \mathrm{CW}$ output of a master oscillator is divided into two signal lines. One of these lines is modulated by a $1 \sim 4 \mu \mathrm{sec}$ pulse width and $60 \mathrm{~Hz}$ repetition rate by a PIN-diode pulse modulator, and the optimum input power of the booster klystron is fed by a $300 \mathrm{~W}$ TWT amplifier. Another line is provided for the reference line through a low-wattage CW amplifier. The output power of the booster klystron is fed into two $2856 \mathrm{MHz}$ prebuncher cavities and a $2856 \mathrm{MHz}$ standing wave 13-cell side couple-type buncher. The klystron drive system has a $70 \mathrm{~m}$ waveguide with directional couplers and feeds into the power attenuator/phase shifter $(\mathrm{I} \phi \mathrm{A})$ placed at each 80 MW klystron. The 13-set klystron is operated in the region from $40 \mathrm{MW}$ to $60 \mathrm{MW}$ in normal operation.

\subsection{Measurement system}

The RF power and phase were measured by using a calibrated crystal detector and a double balanced mixer, respectively. In particular, it was necessary to guarantee the phase stability of the reference line (design value was 0.25 deg. $/{ }^{\circ} \mathrm{C}$ at $70 \mathrm{~m}$ ) with regard to the phase measurement. In order to confirm the high stability at the reference line, the phase was measured by a comparison between the input wave and the reflected wave from the termination of the reference line during one day. This measurement result was $0.8 \mathrm{deg} . / 3.0^{\circ} \mathrm{C}$, which was in agreement with the design value.

The following devices were chosen as the RF equipment for the measurement: the master oscillator, the TWT amplifier, the booster klystron, the drive line at the M14 klystron (about $60 \mathrm{~m}$ from the booster klystron) and the $80 \mathrm{MW}$ klystron (M14 klystron). In this measurement, the RF pulse width was set at $2.2 \mu \mathrm{sec}$. The $2.2 \mu \mathrm{sec}$ pulsed signal from the crystal detectors and the mixers were measured with oscilloscopes whose triggers were synchronized with the beam trigger. All of the oscilloscopes and the data-taking recorders used for temperature measurement were controlled by a sub-control computer (PC) through the GPIB. The data acquisition program was done for all of the oscilloscopes in the stop condition in order to obtain the simultaneity of each RF equipment state and the beam timing. The data acquisition was completed after compensation of the calibration value and attenuation level for the pulse signal from each piece of equipment. This series of measurements was operated routinely every $15 \mathrm{sec}$.

\subsection{Measurement and analysis}

This RF measurement was carried out during the period of one month. The power stability of the master oscillator and the TWT amplifier was less than $0.5 \%$, equal to the accuracy of the measurement, and the phase stability was less than 0.2 deg., equal to the accuracy of the measurement. The long term drift was related to the room temperature, which was controlled so as to take priority of the humidity at the klystron gallery. The phase drift of the high-power klystron drive system, which depends on the environmental temperature, was $10.0 \mathrm{deg} . / 4.0^{\circ} \mathrm{C}$ through one day as shown in Fig. 4.

The high-power RF components including klystron cavities and waveguide had been temperature conditioned within $3.0^{\circ} \mathrm{C}$ by the cooling water except for the accelerating structure, which was controlled by cooling water regulated within $0.1^{\circ} \mathrm{C}$. The power stability for the booster klystron was retained under $0.5 \%$. However, the phase drift that depends on the cooling water temperature was 1.2 deg. $/ 3.0^{\circ} \mathrm{C}$ with a period of 25 minutes. Furthermore, the power and phase drift of the 80MW klystron (M14), which depends on the cooling water temperature, were less than 1.0 MW $/ 3.0^{\circ} \mathrm{C}$ under $50 \mathrm{MW}$ operation and $2.4 \mathrm{deg} . / 3.0^{\circ} \mathrm{C}$ with a period of 25 minutes as shown in Fig. 5.

The power and phase drift of klystron were not only affected by the cooling water temperature, which had a period of 25 minutes, but also by an random variation was caused by fluctuation of the PFN voltage at the $80 \mathrm{MW}$ klystron pulse modulator. Although the PFN voltage of all of the pulse modulators had to be regulated with the stability of $\pm 0.5 \%$ by the de-Qing system, the PFN voltage fluctuation of the M14 pulse modulator was $\pm 1.0 \%$ due to the inadequate adjustment of the de-Qing efficiency. The PFN voltage stability of all the pulse modulators in continuous operation of 12 hours is given in Table 1 . 

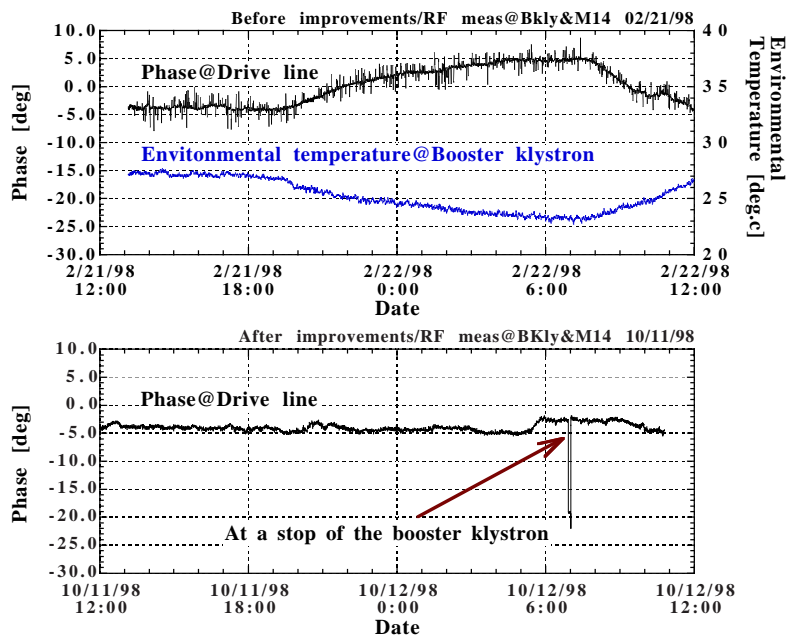

Figure 4: Phase drift for the drive line at M14 I $\phi \mathrm{A}$
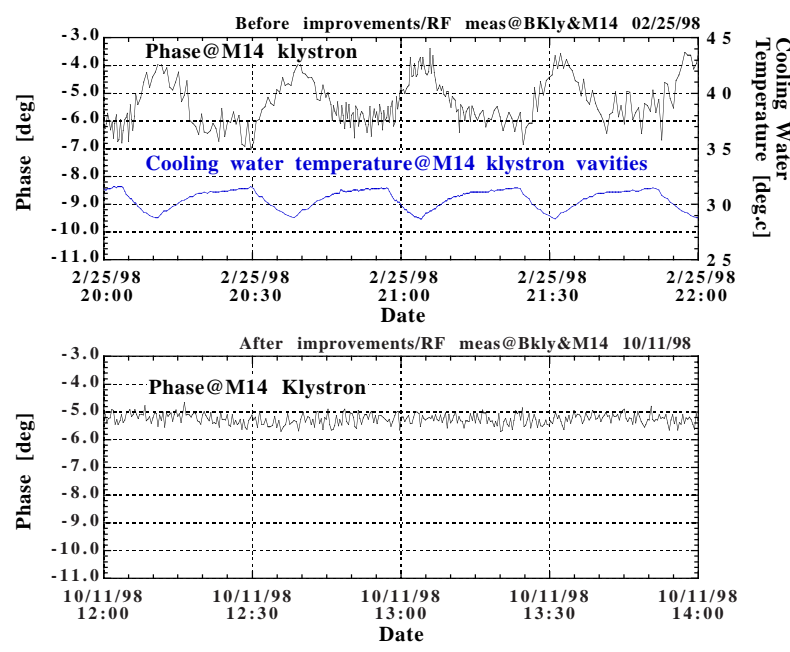

Figure 5: Phase drift for the 80 MW klystron (M14)

\subsection{Results after improvements}

In order to reduce the long term phase drift of the highpower klystron drive system, its $70 \mathrm{~m}$ waveguide was covered with a heat insulator. In addition, the priority of humidity control was replaced by the priority of room temperature control in the klystron gallery. After these improvements, the phase drift of the high-power klystron drive system was achieved at levels smaller than $3.0 \mathrm{deg}$. through one day as shown in Fig. 4. In the cooling water control for klystron cavities, the fan control of the coolant tower had been improved to continuous rotation by using an inverter control from a switching system like an on/off control. After this improvement, the phase stability of the klystrons were reduced to within $0.5 \mathrm{deg}$. as shown in Fig. 5. Readjustment was made to the specified de-Qing efficiency value of $7.0 \%$, so that the PFN voltage stability achieved $0.2 \%(1 \sigma)$ for each klystron as shown in Table 1.

The reproduction and stability of the beam status after the above improvements realized a beam current of $0.7 \%$
Table 1: PFN voltage stability of the pulse modulators (measurement time: 12 hours)

\begin{tabular}{lccc}
\hline & $\begin{array}{c}\text { Klystron } \\
\text { Voltage } \\
(\text { mean)[kV] }\end{array}$ & $\begin{array}{c}\text { Dispersion } \\
(1 \sigma)[\%] \\
\text { Before } \\
\text { adjustment }\end{array}$ & $\begin{array}{c}\text { Dispersion } \\
(1 \sigma)[\%] \\
\text { After } \\
\text { adjustment }\end{array}$ \\
\hline Booster & 137.3 & 0.3 & 0.16 \\
H0 & 310.4 & 1.1 & 0.25 \\
H1 & 337.3 & 0.6 & 0.21 \\
H3 & 334.1 & 0.8 & 0.22 \\
H5 & 353.5 & 0.3 & 0.22 \\
M2 & - & - & - \\
M4 & 338.2 & 0.4 & 0.20 \\
M6 & 351.8 & 0.4 & 0.21 \\
M8 & 350.3 & 0.4 & 0.20 \\
M10 & - & - & - \\
M12 & 337.4 & 0.6 & 0.22 \\
M14 & 325.6 & 1.0 & 0.22 \\
M16 & - & - & - \\
M18 & 364.1 & 0.6 & 0.19 \\
\hline
\end{tabular}

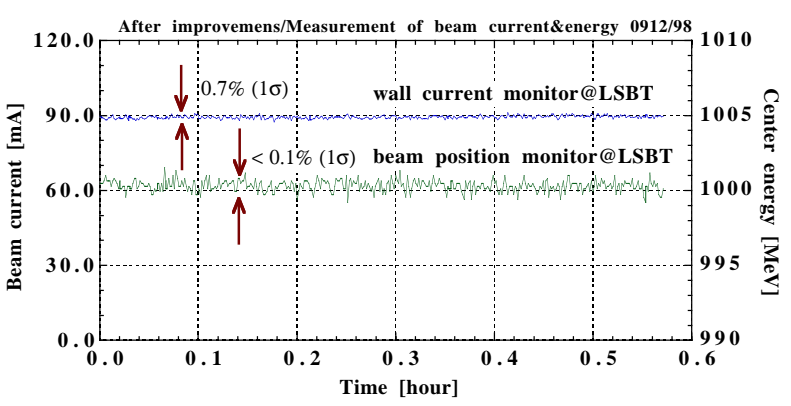

Figure 6: Log data of beam current and center energy

$(1 \sigma)$ and a center energy of $0.1 \%(1 \sigma)$ at the LSBT that was equipped a wall current monitor and a beam position monitor. Figure 6 shows the log data of the beam current and center energy at the LSBT.

\section{CONCLUSION}

In order to realize of the high-stability beam injection into the storage ring, we investigated the cause of the drift of the output power and phase for many type of RF equipment. After making improvements in the utility based on the measurement results of these types of RF equipment, the stability of beam current in LSBT could be maintained within $0.7 \%(1 \sigma)$ without using a energy feedback system.

\section{ACKNOWLEDGEMENTS}

The authors wish to thank M. Adachi and T. Ohnishi of SPring-8 service corporation for beam operation, the data recording, and their cooperation and support.

\section{REFERENCES}

[1] S. Suzuki et al., "Construction of Spring-8 Linac", Proc. of the 4th European Accelerator Conference, London, July 1994 